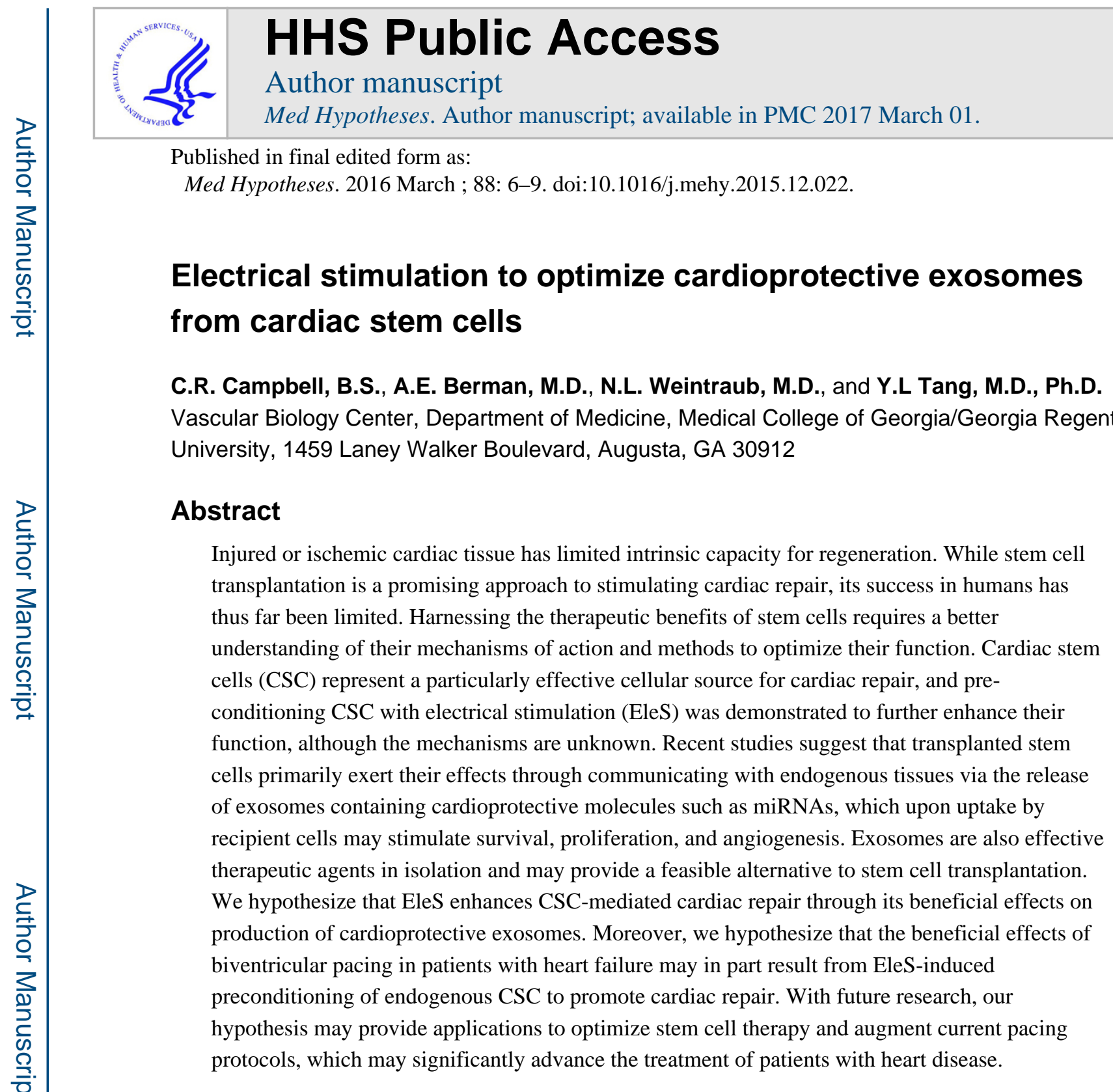

\title{
Introduction
}

Heart failure, commonly precipitated by myocardial infarction (MI), is a leading cause of death in the United States and worldwide. Although various advances in treatment have increased initial survival rates following MI, the irreversible injury to the myocardium results in cardiac dysfunction, remodeling, and ultimately, a high mortality rate, with an estimated 50\% 5-year survival rate in patients post-MI (1). For the patient, ischemic heart disease results in an inevitable decrease in quality of life and an economic burden imposed

Corresponding Author: Y.L. Tang, Tel.: (706) 721-8467, Fax: (706) 721-9799, yaotang@ gru.edu.

Publisher's Disclaimer: This is a PDF file of an unedited manuscript that has been accepted for publication. As a service to our customers we are providing this early version of the manuscript. The manuscript will undergo copyediting, typesetting, and review of the resulting proof before it is published in its final citable form. Please note that during the production process errors may be discovered which could affect the content, and all legal disclaimers that apply to the journal pertain.

Conflict of Interest

None to disclose. 
by healthcare expenses plus the potential loss of income due to illness-related disability. Furthermore, in the United States, the prevalence of cardiovascular disease is expected to increase by nearly $10 \%$ by 2030 (2). In addition to promoting strategies to manage risk factors and prevent cardiovascular disease, treatments to repair the injured heart and restore cardiac function following an ischemic event must be developed and optimized in order to reduce the mortality and morbidity of ischemic heart disease.

\section{Stem cell therapy}

The morbidity and mortality post-MI are in part related to the limited intrinsic capacity of the ischemic heart for regeneration and repair, and one potentially promising approach to promoting cardiac regeneration is stem cell transplantation. While multipotent stem cells specific to tissue type reside in various tissues and serve as a source of repair and renewal in adults, they are generally insufficient to support cardiac repair. This is likely due in part to the fact that the quantity of cardiac stem cells diminishes by up to $40 \%$ post MI, which hampers their ability to induce repair at this critical time of injury (3). Therefore, exogenous stem cells implanted post-MI may supplement the activity of the depleted endogenous cells and stimulate regeneration and repair within the ischemic myocardium.

Implantation of several types of stem cells, including bone marrow derived mesenchymal stem cells (BMC), cardiac stem cells (CSC), and embryonic stem cells have been studied and all, particularly CSC (4), have been demonstrated to improve cardiac function in animal models of MI (5). However, clinical trials using stem cell transplantation in humans post-MI have reported mixed results: for example, one meta-analysis indicated positive long-term outcomes following BMC transplantation, including overall reduction in mortality, reduction in infarct size, and improved left ventricle function (6) while another more recent metaanalysis reported no significant benefit (7). Therefore, further investigations to understand the mechanisms of stem cell action are necessary in order to optimize stem cell therapy and improve clinical efficacy.

\section{Exosomes}

Although the mechanisms of stem cell action post-transplantation remain unclear, research has suggested that survival of transplanted stem cells within the myocardium is limited (8). Stem cells primarily exert their benefit through their communication with endogenous cardiac tissue, which promotes angiogenesis (5) and cardiomyocyte proliferation (9) while decreasing cardiomyocyte apoptosis (5). This communication occurs through exosomes, membrane-bound extracellular vesicles $30-100 \mathrm{~nm}$ in diameter, which interact with surface receptors and exert effects upon internalization within recipient cells $(10,11)$. Barile et al. determined that exosomes are primarily responsible for the anti-apoptotic and proangiogenic effects of cardiac progenitor cells (CPC) transplanted within the ischemic myocardium, as CPC depleted of exosomes produced no cardioprotective effects (12). Additionally, exosomes administered alone may induce cardioprotection and are being examined as an alternative therapeutic strategy to stem cell transplantation (13). Therefore, optimizing the quantity and cardioprotective content of exosomes would represent an important advancement in stem cell research. 
Exosomes transport proteins, signaling molecules, and post-translational modifiers such as miRNAs that may regulate recipient cell function $(10,12,14)$. These molecules are packed into exosomes from the host cell's cytoplasm through ESCRT (endosomal sorting complexes required for transport) or with the help of exosome-specific tetraspanin proteins such as CD63 (10). Because exosome cargo is derived from the host cell's cytoplasmic contents, which may include particles resulting from upregulated survival pathways (15), exosomes convey information about the health of the donor cell (10). Furthermore, because exosomes are a form of cellular communication, exosomes derived from diseased cells, such as platelets in septic patients, may mediate pathological processes, while exosomes derived from stem cells confer transferable protective factors to other cells (11). Exosomes released from mesenchymal stem cells (MSC) were reported to stimulate phosphorylation and activation of the PI3/AKT pathway, resulting in increased survival of the recipient cell (16). Endogenously, exosomes are released constitutively, but their release is increased in response to stress (10). Therefore, exposing stem cells to stress may increase both the quantity and quality of exosomes, which may result in increased benefit to recipient cells.

\section{Pre-conditioning Stem Cells}

Pre-conditioning stem cells before implantation by inducing stress has been demonstrated to enhance their survival and improve their beneficial effects, as demonstrated by changes in exosome contents and function. Activation of AKT and ERK1/2 survival pathways following ischemic preconditioning (exposing cells to cycles of oxygen deprivation) of MSC resulted in increased intracellular levels of miR-210 (15), which has been characterized as an anti-apoptotic exosomal miRNA $(10,13)$. Additionally, exosomes have been reported to transport active pro-survival signaling molecules such as phosphorylated AKT and FAK for direct stimulation of respective pathways in recipient cells (17). Ischemic preconditioning upregulates key pro-survival pathways (14) and results in enhanced exosome signaling. For example, MSC subjected to ischemic preconditioning increased the production and release of exosomal miR-22, which resulted in decreased scar formation in the ischemic myocardium (15). Other methods of pre-conditioning, such as pharmacological agents (18) or genetic modifications (19), do not subject the cells to stress but rather aim to augment the cell's ability to survive within the ischemic myocardium. In devising methods to optimally pre-condition stem cells to survive in the ischemic myocardium, the unique electrical environment of the heart merits consideration.

\section{Electrical Stimulation}

Electrical stimulation (EleS) is a recently-described pre-conditioning method that promotes stem cell survival post-transplantation within the ischemic heart, resulting in improved cardiac function (20). The beneficial effects of EleS may result from induction of cellular stress, similar to ischemic preconditioning, thereby upregulating cytoprotective pathways that enhance their paracrine function. Alternatively, as the heart is constantly exposed to electrical currents, exposing CSC to EleS in vitro, prior to transplantation, may prime the cells to acclimate to the in vivo environment, thereby enhancing their survival and function. In such case, it might be reasoned that perturbation of the electrical currents in the heart, such as occurs in the setting of MI [which is associated with significant impedance changes 
in the area of the infarct (21)], might contribute to the depletion of endogenous CSC that limits the regenerative capacity of the heart post MI (3).

Although the mechanism of EleS remains unclear, pre-conditioning stem cells with electrical stimulation is comparably efficacious to ischemic pre-conditioning in enhancing the beneficial effects of stem cells. However, EleS offered a shorter window of protection than ischemic preconditioning induced by exposure to oxidative stress, suggesting the involvement of different molecular signaling mechanisms (20). Kim et al. demonstrated that pre-conditioning CSC with EleS decreased CSC apoptosis in vivo and in vitro through upregulating the PI3K/AKT and FAK pro-survival pathways. This translated into enhancement of the beneficial effects of stem cell transplantation within the ischemic myocardium (20). Because exosomes convey the cardioprotective effects of stem cells, EleS might have favorably altered exosome content and release. However, the study by Kim et al. did not investigate the impact of EleS on exosomes, so this hypothesis remains to be tested.

Further support for the use of EleS of CSC may be suggested by the positive response of the dysfunctional heart to electrical stimulation. Electrical stimulation of cardiac tissue in the form of biventricular cardiac pacing is an established, effective treatment for reducing the mortality of heart failure (22). Particularly, biventricular pacing has been suggested to improve heart function by reducing cardiac dyssynchrony and reversing maladaptive remodeling, preventing pathological dilation of the left ventricle and progressive dysfunction (22). The mechanisms whereby electrical stimulation improves function at the cellular and molecular level are not yet understood. The favorable effects of EleS on CSC in vitro raises the possibility that optimization of cardiac pacing could activate CSC in vivo, which in turn might promote cardiac repair. Investigating how CSC respond to electrical stimulation may lead to a) optimization of current pacing protocols to better activate endogenous CSC and b) improvements in the therapeutic benefit of stem cell transplantation.

\section{Conclusions}

In conclusion, the purpose of the study is to optimize treatment for ischemic heart failure in order to reduce its mortality and morbidity. Stem cells are a promising tool for regeneration of cardiac tissue, but methodology must be devised to better harness their therapeutic benefit and clinical applicability. Electrical stimulation through biventricular pacing has been demonstrated to improve cardiac function (22), and CSC respond favorably to EleS. Preconditioning CSC with EleS results in activation of pro-survival pathways, and upon transplantation into the ischemic myocardium, EleS-stimulated CSC elicit greater cardiac benefit (20). The cardioprotective effects of stem cells may be primarily accounted for by the release of exosomes with biologically active miRNAs and proteins (12), and exosomes provide therapeutic benefit in isolation (13). Therefore, electrically-stimulated CSC likely release more exosomes with a higher content of protective molecules that promote cardiomyocyte survival and angiogenesis. 


\section{Hypothesis}

We hypothesize that electrical stimulation upregulates the production and bioactivity of cardioprotective exosomes in CSC, thereby boosting their therapeutic potential to elicit cardiac repair.

\section{Discussion}

Our hypothesis introduces novel concepts to the field of cardiovascular stem cell research, so testing the validity of our predictions must occur at the cellular level. First, protocols for EleS as a form of pre-conditioning stem cells pre-implantation must be established and evaluated for efficacy. Kim et al. first used EleS to pre-condition cells for the purpose of increasing the survival of transplanted CSC and enhancing their benefit to the ischemic heart (20). However, EleS has been used to promote the differentiation of CSC (23) and adiposederived stem cells (24) into cardiomyocyte-type cells. Primarily, the difference between electrically pre-conditioning stem cells and inducing their differentiation may depend on the duration of EleS, as pre-conditioning was elicited by short-term EleS (3 hours) (20), while differentiation required long-term stimulation (14 days) (23). However, comprehensive EleS protocols will be required to establish optimal pre-conditioning of CSC. The conditions established by Kim et al. will be replicated using an IonOptix C-Pace EP cell culture stimulator, varying the amplitude, frequency and duration of the stimulus. The EleS parameters will be initially evaluated for efficacy by quantifying the expression of prosurvival genes and phosphorylated AKT in CSC. This "high throughput" methodology will enable us to identify the most promising EleS parameters, which will then be further screened for efficacy by determining their impact on CSC survival in response to noxious stimuli (i.e., oxidative stress, pro-inflammatory cytokines).

Once EleS protocols have been optimized to promote CSC survival, exosomes will be isolated from the medium post EleS conditioning using PureExo® Exosome Isolation Kits. Exosome morphology will be examined by electron microscopy and quantified by nanoparticle tracking analysis. RNA will be isolated from the exosomes, and expression of miRNAs will be quantified by microarray analysis and validated by RT-PCR. Proteins will also be isolated and analyzed using Western Blot. Morphology, quantity, and miRNA and protein content will be quantified and compared to exosomes derived from unstimulated CSC. If our hypothesis is correct, CSC exposed to EleS will release more exosomes with significantly higher levels of prosurvival proteins, such as phosphorylated AKT and FAK (20) and cardioprotective miRNAs, such as miR-210 and miR-132 (25), and reduced expression of miRNAs that have been linked to cell death, adhesion, and inflammation, such as $\mathrm{miR}-378(20)$.

If EleS favorably impacts the exosomal miRNA and protein expression profile in CSC, purified exosomes will be collected, labeled and applied to cultured cardiomyocytes and endothelial cells to determine the impact on $\mathrm{H}_{2} \mathrm{O}_{2}$-induced cardiomyocyte apoptosis and angiogenesis, respectively, using standard methods. If exosomes from CSC exposed to EleS significantly reduce cardiomyocyte apoptosis and promote angiogenesis in vitro, we would proceed to test them in vivo using a mouse model of myocardial infarction. If successful, this 
protocol may be developed further in animal models and eventually adapted for testing in humans.

Exosomes derived from stem cells are a promising therapeutic tool, and although they may pose less risk than cellular transplantation, challenges remain in terms of optimizing their stability and application. Exosomes are unstable and prone to aggregate with a measured zeta potential of -14 to $-15 \mathrm{mV}$ at $23^{\circ} \mathrm{C}$ (13), but stability may be increased at physiological body temperature, and recent clinical trials have demonstrated that isolated exosomes may be feasible as therapeutic agents in humans when used in an anti-cancer vaccine for stage IV melanoma (26). While exosomes have shown promise in animal models of cardiac ischemia (13), methods to optimally deliver exosomes into the human heart have yet to be established, and the long-term effects have not been studied. We speculate that preconditioning with EleS will enhance both the quantity and quality of exosomes released by CSC, without adversely impacting safety, thus enhancing the efficacy of cardiac repair in humans.

Assessing the benefits of EleS in CPC may provide insight into how to best activate endogenous CPC to promote cardiac recovery in patients with heart failure. Electrical stimulation through biventricular pacing has been demonstrated to improve heart function in subsets of patients, and it is possible that this benefit may in part be mediated by the effects of EleS on endogenous CSC. Therefore, if successful, our pacing protocols developed in vitro may be applied clinically in patients with biventricular pacemakers to determine whether modifying stimulus amplitude, frequency and duration can improve heart function, and favorably impact the release of cardiac miRNAs into the blood, as compared to standard biventricular pacing protocols.

\section{Implications}

Development of an electrical stimulation protocol as a form of pre-conditioning CSC will help to advance our understanding of the mechanisms whereby stem cells produce beneficial effects in patients with heart failure. Moreover, this research may lead to high-throughput methodology to optimize the quantity and quality of exosomes produced by exogenous CSC for therapeutic use in humans. Importantly, the electrical stimulation protocol may then be applied to patients with biventricular pacemakers to determine whether it can stimulate endogenous CSC to enhance recovery of heart function. Therefore, with further research, our hypothesis may have implications to optimize the future treatment and management of ischemic heart disease and alleviate the growing epidemic of heart failure worldwide.

\section{Acknowledgments}

Support: This work was supported by the American Heart Association Beginning Grant-in-Aid 0765094Y (to Y.T.); NIH Grant HL086555 (to Y.T.), and NIH Grants HL076684 and HL62984 (to N.L.W.); GRU Medical Scholars Program (to C.R.C.)

\section{References}

1. Law MR, Watt HC, Wald NJ. The Underlying Risk of Death After Myocardial Infarction in the Absene of Treatment. Arch Intern Med. 2002; 162(21):2405-2410. [PubMed: 12437397] 
2. Heidenreich PA, Trogdon JG, Khavjou OA, et al. Forecasting the future of cardiovascular disease in the United States: a policy statement from the American Heart Association. Circulation. 2011; 123(8):933-44. [PubMed: 21262990]

3. Mouquet F, Pfister O, Jain M, et al. Restoration of cardiac progenitor cells after myocardial infarction by self-proliferation and selective homing of bone marrow-derived stem cells. Circ Res. 2005; 97(11):1090-2. [PubMed: 16269652]

4. Tang YL, Wang YJ, Chen LJ, Pan YH, Zhang L, Weintraub NL. Cardiac-derived stem cell-based therapy for heart failure: progress and clinical applications. Exp Biol Med. 2013; 238(3):294-300.

5. Segers VF, Lee RT. Stem-cell therapy for cardiac disease. Nature. 2008; 451(7181):937-42. [PubMed: 18288183]

6. Jeevanantham V, Butler M, Saad A, Abdel-Latif A, Zuba-Surma EK, Dawn B. Adult bone marrow cell therapy improves survival and induces long-term improvement in cardiac parameters: a systematic review and meta-analysis. Circulation. 2012; 126(5):551-68. [PubMed: 22730444]

7. Gyongyosi M, Wojakowski W, Lemarchand P, et al. Meta-Analysis of Cell-based CaRdiac stUdiEs (ACCRUE) in patients with acute myocardial infarction based on individual patient data. Circ Res. 2015; 116(8):1346-60. [PubMed: 25700037]

8. Liao S-Y, Tse H-F. Multipotent (adult) and pluripotent stem cells for heart regeneration: what are the pros and cons? Stem Cell Res Ther. 2013; 4(6):151. [PubMed: 24476362]

9. Khan M, Nickoloff E, Abramova T, et al. Embryonic Stem Cell-Derived Exosomes Promote Endogenous Repair Mechanisms and Enhance Cardiac Function Following Myocardial Infarction. Circ Res. 2015; 117(1):52-64. [PubMed: 25904597]

10. Emanueli C, Shearn AI, Angelini GD, Sahoo S. Exosomes and exosomal miRNAs in cardiovascular protection and repair. Vascul Pharmacol. 2015

11. Ailawadi S, Wang X, Gu H, Fan GC. Pathologic function and therapeutic potential of exosomes in cardiovascular disease. Biochim Biophys Acta. 2015; 1852(1):1-11. [PubMed: 25463630]

12. Barile L, Lionetti V, Cervio E, et al. Extracellular vesicles from human cardiac progenitor cells inhibit cardiomyocyte apoptosis and improve cardiac function after myocardial infarction. Cardiovasc Res. 2014; 103(4):530-41. [PubMed: 25016614]

13. Wang Y, Zhang L, Li Y, et al. Exosomes/microvesicles from induced pluripotent stem cells deliver cardioprotective miRNAs and prevent cardiomyocyte apoptosis in the ischemic myocardium. Int $\mathbf{J}$ Cardiol. 2015; 192:61-9. [PubMed: 26000464]

14. Lai RC, Arslan F, Lee MM, et al. Exosome secreted by MSC reduces myocardial ischemia/ reperfusion injury. Stem Cell Res. 2010; 4(3):214-22. [PubMed: 20138817]

15. Kim HW, Haider HK, Jiang S, Ashraf M. Ischemic preconditioning augments survival of stem cells via miR-210 expression by targeting caspase-8-associated protein 2. J Biol Chem. 2009; 284(48):33161-8. [PubMed: 19721136]

16. Arslan F, Lai RC, Smeets MB, et al. Mesenchymal stem cell-derived exosomes increase ATP levels, decrease oxidative stress and activate PI3K/Akt pathway to enhance myocardial viability and prevent adverse remodeling after myocardial ischemia/reperfusion injury. Stem Cell Res. 2013; 10(3):301-12. [PubMed: 23399448]

17. Biasutto L, Chiechi A, Couch R, Liotta LA, Espina V. Retinal Pigment Epithelium (RPE) exosomes contain signaling phosphoproteins affected by oxidative stress. Exp Cell Res. 2013; 319(13):2113-2123. [PubMed: 23669273]

18. Hoke NN, Salloum FN, Kass DA, Das A, Kukreja RC. Preconditioning by phosphodiesterase-5 inhibition improves therapeutic efficacy of adipose-derived stem cells following myocardial infarction in mice. Stem Cells. 2012; 30(2):326-35. [PubMed: 22102597]

19. Hahn J, Cho H, Bae J, Yuk H, Kim K, Park K. Beta-catenin overexpression reduces myocardial infarct size through differential effects on cardiomyocytes and cardiac fibroblasts. J Biol Chem. 2006; 281:30979-89. [PubMed: 16920707]

20. Kim SW, Kim HW, Huang W, et al. Cardiac stem cells with electrical stimulation improve ischaemic heart function through regulation of connective tissue growth factor and miR-378. Cardiovasc Res. 2013; 100(2):241-51. [PubMed: 24067999] 
21. Fallert MA, Mirotznik MS, Downing SW, et al. Myocardial electrical impedance mapping of ischemic sheep hearts and healing aneurysms. Circulation. 1993; 87(1):199-207. [PubMed: 8419008]

22. Jaffe LM, Morin DP. Cardiac Resynchronization Therapy: History, Present Status, and Future Directions. Ochsner J. 2014; 14(4):596-607. [PubMed: 25598725]

23. Llucià-Valldeperas A, Sanchez B, Soler-Botija C, et al. Physiological conditioning by electric field stimulation promotes cardiomyogenic gene expression in human cardiomyocyte progenitor cells. Stem Cell Res Ther. 2014; 5(4):93. [PubMed: 25092238]

24. Pavesi A, Soncini M, Zamperone A, et al. Electrical conditioning of adipose-derived stem cells in a multi-chamber culture platform. Biotechnol Bioeng. 2014; 111(7):1452-63. [PubMed: 24473977]

25. Das S, Halushka MK. Extracellular vesicle microRNA transfer in cardiovascular disease. Cardiovasc Pathol. 2015

26. Chaput N, Taieb J, Schartz N, et al. The potential of exosomes in immunotherapy of cancer. Blood Cell Mol Dis. 2005; 35(2):111-5. 